\title{
Single-Cell RNA Sequencing in Tumor-Infiltrating T Cells Research
}

\author{
Zhenchuan $\mathrm{Wu}^{*}$ \\ CAS Key Laboratory of Tissue Microenvironment and Tumor, Shanghai Institute of Nutrition and Health, Shanghai Institutes for \\ Biological Sciences, University of Chinese Academy of Sciences, China
}

*Corresponding author: Zhenchuan Wu, CAS Key Laboratory of Tissue Microenvironment and Tumor, Shanghai Institute of Nutrition and Health, Shanghai Institutes for Biological Sciences, University of Chinese Academy of Sciences, China

\begin{tabular}{|c|c|}
\hline ARTICLE INFO & ABSTRACT \\
\hline Received: 蔧 November 22, 2019 & Citation: Zhenchuan Wu. Single-Cell RNA Sequencing in Tumor-Infiltrating T Cells \\
\hline Published: 櫘 December 02, 2019 & Research. Biomed J Sci \& Tech Res 23(3)-2019. BJSTR. MS.ID.003902. \\
\hline
\end{tabular}

\section{Introduction}

During the development of next-generation sequencing, RNA sequencing has become an indispensable deep-sequencing technology for measurement of levels of transcripts and isoforms [1]. But traditional RNA-seq from tissue and/or cells cannot easily resolve specific cell types. Now, exciting new applications are being explored, RNA-seq meet the greatly chance for its new technology that is single-cell RNA sequencing(scRNA-seq). Unlike traditional bulk RNA sequencing analyzing gene expression in bulk level, single-cell RNA sequencing can detect the global transcriptome of thousands of isolated cells on single cell level [2]. The applications of single-cell sequencing are widely, such as cancer research, developmental biology [3] and neurosciences [4]. In this review, we focus on the application of scRNA-seq on tumor-infiltrating T cells research. scRNA-seq can give us a map of tumor microenvironment by decomposition of complex tumor tissues into functionally distinct cell types and reveal cell types that are unknown in what were considered well-studied tumor diseases. scRNA-seq increase our understanding of the tumor-infiltrating $\mathrm{T}$ cells and potentially help us identify new immunotherapy targets.

The steps of scRNA-seq method can borrow from earlier bulk RNA-seq protocols. Single lymphocytes can isolate from peripheral blood, tumor, and adjacent normal tissues from patients. Most labs have access to flow-cytometry instrumentation and use microtiter plates containing lysis buffer [5]. For higher-throughput experiments can refer to droplet-microfluidic isolation, such as Drop-Seq [6] or InDrop [5]. Each single cell that tagged with Unique Molecular Identifiers (UMIs) is reverse transcribed in order to produce
cDNA, and the cDNA is used as the input for RNA-seq library preparation. The transcriptional profiles of these individual cells, coupled with assembled T cell receptor (TCR), are sequenced [7]. Using unsupervised algorithms to cluster cell types and then assigned to cell types according to aggregated cluster-level expression profiles and delineate their developmental trajectory [8]. Most study may focus on analyze the cell-type composition and study dynamics of mixed cell population in various biological contexts. Tumor-infiltrating lymphocytes are highly heterogeneous, because of a variety of compositions of cell-type and gene expression profiles on tumor microenvironment. T cell patterns are distinct in both tumors and adjacent normal tissue. Yannick Simoni et al. reported that in tumor microenvironment CD8+ T cells are phenotypically heterogeneous within a tumor and across patients, and bystander CD8+ T cells are abundant and distinct in human tumor infiltrates [9].

The state of tumor-infiltrating $\mathrm{T}$ cells can be divided into cytotoxic, bystander cytotoxic, exhausted and dysfunctional state. The functional of different state $\mathrm{T}$ cells within tumors remain unknown. Analysis of paired single-cell RNA and T cell receptor sequencing data, Hanjie Li et al. reported a gradient of dysfunctional $\mathrm{T}$ cell are associated with tumor reactivity and are the major intratumoral proliferating immune cell compartment on melanoma [10]. Tirosh et al. revealed $\mathrm{T}$ cell exhaustion signature may connect to $\mathrm{T}$ cell activation and clonal expansion on melanoma turmors [11]. These findings provide evidence that dysfunctional $\mathrm{T}$ cells may be a driver of tumor reactive, equally to cytotoxic T cells. Tumor microenvironment have differential impact on $\mathrm{T}$ cell dysfunction across tumor 
types. We need scRNA-seq to describe tumor infiltrates. The transcriptomes of $\mathrm{T}$ cell subset help to identify previously unknown marker for prognosis. For example, Xinyi Guo et al. reported a ratio of pre-exhausted to exhausted $\mathrm{T}$ cells are relative to better prognosis of lung adenocarcinoma [12]. Peter Savas et al. demonstrated that tumor-infiltrating lymphocytes in breast cancer contains several CD8+ T cells with features of tissue-resident memory expressing high levels of immune checkpoint molecules and effector proteins, which are associated with good prognosis in breast cancer [13]. Chuanhong zheng et al. reported primary CD8+ T cells over-expressing LAYN results in inhibition of interferon-gama production, which suggesting LAYN is linked to the suppressive function of tumor Treg and exhausted CD8 T cells [14]. Overall, these findings provided an exciting vision of how we use scRNA-seq to discover tumor immune markers and understand their roles in regulating immune response and tissue-specific functions.

The development and migration of T cells within tumors remain unknown. scRNA-seq has also been instrumental in resolving details of the trajectory and regulation of T cells. T Cell Receptor (TCR) clonotypes determine the developmental trajectories of $\mathrm{T}$ cells and reveal phenotypic diversity. Tumor antigen specific TCR clusters also are key components in anti-tumor immune response. scRNAseq of TCR gene repertoires are useful for to reveal the intrinsic heterogeneity among antigen-specific $\mathrm{T}$ cells and their function in tumor response [7]. David Redmond et al. reported a method to identification and assembly of full-length rearranged V(D)J T cell receptor sequences from scRNA-seq data [15]. Combined with TCR analysis, EIham Azizi et al. yielded an immune map of breast cancer that points to continuous $\mathrm{T}$ cell activation and differentiation trajectories [16]. In short, identifying clonal TCRs at single-cell levels allows us to discover their developmental trajectory in various $\mathrm{T}$ cell clusters, and deduce their activation status in tumor microenvironment.

In conclusion, scRNA-seq measures the expression levels of genes in cells in a comprehensive, sensitive and accurate way. scRNA-seq is aiding in the discovery of the heterogeneity of tumorinfiltrating lymphocytes, unreported subpopulations and states, potential biomarkers, tumor antigen-specific TCR clusters and their relationship to physiology and disease.

\section{Acknowledgement}

The author acknowledges support from the National Key Research and Development Program of China (2017YFA0103501,
2016YFC1305502) and the Chinese Academy of Sciences (XDA12010203, QYZDJ-SSW-SMC017).

\section{References}

1. Z Wang, M Gerstein, M Snyder (2009) RNA-Seq: a revolutionary tool for transcriptomics. Nat Rev Genet 10(1): 57-63.

2. AA Kolodziejczyk, J K Kim, V Svensson, J C Marioni, SA Teichmann (2015) The technology and biology of single-cell RNA sequencing. Mol Cell 58(4): 610-620.

3. SS Potter (2018) Single-cell RNA sequencing for the study of development, physiology and disease. Nat Rev Nephrol 14(8): 479-492.

4. D Ofengeim, N Giagtzoglou, D Huh, C Zou, J Yuan (2017) Single-Cell RNA Sequencing: Unraveling the Brain One Cell at a Time. Trends Mol Med 23(6): 563-576.

5. C Ziegenhain, Vieth B, Parekh S, Reinius B, Guillaumet Adkins A, et al. (2017) Comparative Analysis of Single-Cell RNA Sequencing Methods. Mol Cell 65(4): 631-643.

6. J Bageritz and G Raddi (2019) Single-Cell RNA Sequencing with DropSeq. Methods Mol Biol 1979: 73-85

7. A Han, J Glanville, L Hansmann, MM Davis (2014) Linking T-cell receptor sequence to functional phenotype at the single-cell level. Nat Biotechnol. 32(7): 684-692

8. AA AlJanahi, M Danielsen, CE Dunbar (2018) An Introduction to the Analysis of Single-Cell RNA-Sequencing Data. Mol Ther Methods Clin Dev 10: 189-196.

9. Y Simoni, Becht E, Fehlings M, Loh CY, Koo SL, et al. (2018) Bystander CD8+ T cells are abundant and phenotypically distinct in human tumour infiltrates. Nature 557(7706): 575-579.

10. H Li, van der Leun AM, Yofe I, Lubling Y, Gelbard Solodkin D, et al. (2019) Dysfunctional CD8 T Cells Form a Proliferative, Dynamically Regulated Compartment within Human Melanoma. Cell 176(4): 775-789.

11. I Tirosh, Izar B, Prakadan SM, Wadsworth MH, Treacy D, et al. (2016) Dissecting the multicellular ecosystem of metastatic melanoma by single-cell RNA-seq. Science 352(6282): 189-196.

12.X Guo, Zhang Y, Zheng L, Zheng C, Song J, et al. (2018) Global characterization of $\mathrm{T}$ cells in non-small-cell lung cancer by single-cell sequencing. Nat Med 24(7): 978-985.

13. P Savas, Virassamy B, Ye C, Salim A, Mintoff CP, et al. (2018) Singlecell profiling of breast cancer $\mathrm{T}$ cells reveals a tissue-resident memory subset associated with improved prognosis. Nat Med 24(7): 986-993.

14. C Zheng, Zheng L, Yoo JK, Guo H, Zhang Y, et al. (2017) Landscape of Infiltrating $\mathrm{T}$ Cells in Liver Cancer Revealed by Single-Cell Sequencing. Cell 169(7): 1342-1356.

15. D Redmond, A Poran, and O Elemento (2016) Single-cell TCRseq: paired recovery of entire T-cell alpha and beta chain transcripts in T-cell receptors from single-cell RNAseq. Genome Med 8: 80.

16. E Azizi, Carr AJ, Plitas G, Cornish AE, Konopacki C, et al. (2018) Single-Cell Map of Diverse Immune Phenotypes in the Breast Tumor Microenvironment. Cell 174(5): 1293-1308. 


\section{ISSN: 2574-1241}

DOI: 10.26717/BJSTR.2019.23.003902

Zhenchuan Wu. Biomed J Sci \& Tech Res

(C) (P) This work is licensed under Creative

Submission Link: https://biomedres.us/submit-manuscript.php

$\begin{array}{ll}\text { BIOMEDICAL } & \text { Assets of Publishing with us } \\ \text { RESEARCHES } & \text { - Global archiving of articles } \\ \text { - Immediate, unrestricted online access } & \text { - Rigorous Peer Review Process } \\ & \text { - Authors Retain Copyrights } \\ & \end{array}$

